\title{
ON A THEOREM OF STEINITZ AND LEVY
}

\author{
BY
}

\section{GADI MORAN}

\begin{abstract}
Let $\sum_{n \in \omega} h(n)$ be a conditionally convergent series in a real Banach space $B$. Let $S(h)$ denote the set of sums of the convergent rearrangements of this series. A well-known theorem of Riemann states that $S(h)=B$ if $B=R$, the reals. A generalization of Riemann's Theorem, due independently to Levy [L] and Steinitz [S], states that if $B$ is finite dimensional, then $S(h)$ is a linear manifold in $B$ of dimension $>0$. Another generalization of Riemann's Theorem [M] can be stated as an instance of the Levy-Steinitz Theorem in the Banach space of regulated real functions on the unit interval $I$. This instance generalizes to the Banach space of regulated $B$-valued functions on $I$, where $B$ is finite dimensional, implying a generalization of the Levy-Steinitz Theorem.
\end{abstract}

1. Let $\omega=\{0,1,2, \ldots\}$ denote the set of natural numbers, $B$ a real Banach space, and let $h$ be a function from $\omega$ into $B$. We say that the series $\Sigma_{n \in \omega} h(n)$ is conditionally convergent if there are two rearrangements of its terms, one resulting in a convergent series and the other a divergent one. It is unconditionally convergent if it is convergent for every rearrangement of its terms. If the series is conditionally (unconditionally) convergent we say that $h$ is conditionally (unconditionally) summable.

Let $S(h)$ denote the set of those $v$ in $B$ that are sums (in the norm) of some convergent rearrangement of $\sum_{n \in \omega} h(n)$. It is well known, that if $h$ is unconditionally summable then $S(h)$ has precisely one member. ${ }^{1}$

A hundred years ago Riemann showed:

THEOREM 1. Let $B=R$. If $h: \omega \rightarrow B$ is conditionally convergent, then $S(h)=R$.

Levy-Steinitz's Theorem (1905) generalizes as follows:

THEOREM 2. Let $B$ be finite dimensional. ${ }^{2}$ If $h: \omega \rightarrow B$ is conditionally

Received by the editors May 26, 1977.

AMS (MOS) subject classifications (1970). Primary 40A99, 46B15; Secondary 46E15.

Key words and phrases. Conditionally convergent series, rearrangement of terms, finite dimensional Banach space.

${ }^{1} \mathrm{See}$ [H], [O]. The converse is true if $B$ is finite dimensional, but fails already in any infinite dimensional Hilbert space, as the example following Theorem 2 indicates.

2 The proofs of Theorem 2 [L], [S], [B1] assume the Euclidean norm. Since all norms induce the same topology on $R^{n}, S(h)$ is independent of the particular norm chosen.

(c) American Mathematical Society 1979 
summable, then $B$ has a subspace $N$ of dimension $>0$ and a member $v_{0}$ such that

$$
S(h)=v_{0}+N .
$$

It is easy to construct an $h$ from $\omega$ into any inifinite dimensional Hilbert space such that $S(h)=\{0\}$, but $h$ is not unconditionally convergent (let $e_{n}$ be an orthonormal sequence, and let $h(2 n)=e_{n} / \sqrt{n+1}=-h(2 n+1)$ ). Thus, Theorem 2 does not generalize to infinite dimensional Banach spaces. (See, however [D1].) It is a long standing conjecture that for arbitrary Banach space $B$ and conditionally summable $h$ from $\omega$ into $B, S(h)$ is a displacement of a closed additive subgroup of $B$.

Another generalization of Theorem 1 emerged from quite a different course of research ([M], see also [G]). It implies that if $h: \omega \rightarrow R$ is conditionally summable, then there is a chain of conditionally convergent subseries of $s=\Sigma_{n \in \omega} h(n)$, order isomorphic to the reals, such that any change in the list of their sums subject to some natural continuity restrictions is achieved by one rearrangement of the series $s$. A precise statement of this result is given in $\$ 2$ as Theorem 3. We now describe it in a way that clarifies its relation with Theorem 2.

Let $I$ denote the closed unit interval $[0,1]$, and let $C(I, B)$ (respectively $\operatorname{Reg}(I, B)$ ) denote the Banach space of all continuous functions (respectively the functions having left and right limit everywhere) from $I$ into $B$, endowed with the supremum norm. For $0<x<1, v \in B$ define $J_{x}^{v} \in \operatorname{Reg}(I, B)$ by

$$
\begin{array}{ll}
J_{x}^{\nu}(t)=0, & t<x, \\
J_{x}^{v}(t)=v, & x<t .
\end{array}
$$

Call a sequence $x=\left(x_{n}\right)_{n \in \omega}$ a dense sequence in $I$ if $x$ enumerates a dense subset of the open interval $(0,1)$ with no repetitions. In the sequel, let $x$ be a fixed dense sequence in $I$. With every $h: \omega \rightarrow B$ we associate $h^{x}: \omega \rightarrow$ $\operatorname{Reg}(I, B)$ by setting $h^{x}(n)=J_{x_{n}}^{h(n)}$. The generalization of Theorem 1 to the present context depends on a proper notion of "conditionality" for $h$. We define it first in case $B=R$.

We call $h: \omega \rightarrow R x$-conditional iff:

(1) for every $\varepsilon>0,\{n:\|h(n)\|>\varepsilon\}$ is finite,

(2) for every $0 \leqslant a<b \leqslant 1$, we have

$$
\begin{aligned}
\sum\{h(n): & \left.a<x_{n}<b, h(n)>0\right\} \\
= & -\sum\left\{h(n): a<x_{n}<b, h(n)<0\right\}=\infty .
\end{aligned}
$$

Clearly if $h$ is $x$-conditional, it is conditionally summable. It follows from [G, Theorem 3], that $h^{x}$ is also conditionally summable. 
Theorem 3 [M]. Let $B=R$, and let $h: \omega \rightarrow B$ be $x$-conditional. Then there is an $s_{0} \in \operatorname{Reg}(I, B)$ such that

$$
S\left(h^{x}\right)=s_{0}+C(I, B) .
$$

Stated this way, Theorem 3 is an instance of Theorem 2 in the infinite dimensional Banach space $\operatorname{Reg}(I, R)$. In $\$ 3$ it is generalized as an instance of Theorem 2 in $\operatorname{Reg}(I, B)$, where $B$ is finite dimensional (Theorem 4). In $\$ 4$ we derive Theorem 5 , which is another generalization of Theorem 1, from Theorem 4. Theorem 5 is then combined with Steinitz's work [S] to give the main result of this paper, Theorem 6, extending Theorem 2.

It is well known that the convergence of every subseries of a series in a Banach space is equivalent to its unconditional convergence (see e.g. [H]), and so every subseries of such a series is also unconditionally convergent, hence has one sum. Theorem 6 implies, by a way of contrast, that a conditionally convergent series in a finite dimensional Banach space $B$ admits a nontrivial subspace $N \subseteq B$ and a chain of (conditionally convergent) subseries, order isomorphic to the reals, such that every continuous change in $N$ of the sums of those subseries is accomplished by some rearrangement of the terms of the given series (Theorem $6^{\prime}$ ). We now turn to make these remarks precise.

2. We develop first some notation. Let $\prec$ be an $\omega$-ordering of $\omega$, i.e. a linear ordering of $\omega$ every intiial segment of which is finite. We denote by $n \prec$ the $n$th member of $\omega$ under $\prec$, and for $C \subseteq \omega$ we set

$$
\stackrel{<, n}{C}=C \cap\left\{0^{\prec}, 1^{\prec}, \ldots,(n-1)^{\prec}\right\} .
$$

$[A]^{<\omega}$ denotes the family of all finite subsets of the set $A$. If $h: \omega \rightarrow B$, $F \in[\omega]^{<\omega}$ we set $h(F)=\sum_{n \in F} h(n)$.

We say that the $\omega$-ordering $\prec$ sums $h$ over $C \subseteq \omega$ if the sequence

$$
v_{n}=h(\stackrel{<, n}{C})
$$

has a limit in $B$. If $\prec$ sums $h$ over $C$ we write

$$
\sum_{n \in C}^{\prec} h(n)=\sum_{C}^{\prec} h=\lim _{n \rightarrow \infty} h(\stackrel{<, n}{C}) \text {. }
$$

Whenever $\prec$ is the natural ordering of $\omega$ we omit it from the notation.

Let $C$ be a family of subsets of $\omega$. We say that $\prec$ sums $h$ over $C$ iff for every $C \in \mathrm{C}, \prec$ sums $h$ over $C$. We say that $\prec$ sums $h$ uniformly over $C$ iff for every $\varepsilon>0$ there is an $n \in \omega$ such that for every $C \in \mathrm{C}, n<k, l$ we have:

$$
\|h(\stackrel{<, k}{C})-h(\stackrel{<, l}{C})\|<\varepsilon .
$$


Thus, $h$ is unconditionally summable if and only if the natural order sums $h$ over $P(\omega)$, the set of all subsets of $\omega$ (by the equivalence of subseries convergence and unconditional convergence). We leave to the interested reader the verification that if $h$ is unconditionally summable, then the natural order sums $h$ uniformly over $P(\omega)$, as does every other $\omega$-ordering of $\omega$, and that if any $\omega$-ordering sums $h$ over $P(\omega)$ then $h$ is unconditionally summable.

We call $C \subseteq P(\omega)$ a chain iff for any $C_{1}, C_{2} \in \mathrm{C}$ we have $C_{1} \subseteq C_{2}$ or $C_{2} \subseteq C_{1}$. We consider a chain $C$ as a linearly ordered set, with set-inclusion as the order. Every chain is order isomorphic to a subset of $I$ (Proof. Let $g(n)=2^{-(n+1)}$. Then $C \rightarrow \Sigma_{C} g$ is an order isomorphism of C into I.) Conversely, if $T$ is a subset of $I$, then there is a chain $C$ in $P(\omega)$ order isomorphic to $T$.

An $I$-chain is an indexed chain $C=\left\{C_{t}: t \in I\right\}$ satisfying $C_{0}=\varnothing, C_{1}=\omega$ and $C_{a} \subsetneq C_{b}$ whenever $0<a<b<1$.

Assume now that $x=\left(x_{n}\right)_{n \in \omega}$ is a dense sequence in $I$. For $t \in I$ let $C_{t}=\left\{n \in \omega: x_{n}<t\right\}$. Then $\mathrm{C}=\left\{C_{t}: t \in I\right\}$ is an I-chain.

Let $h: \omega \rightarrow B, h^{x}: \omega \rightarrow \operatorname{Reg}(I, B)$ be as in $\$ 1$. If for some $\omega$-ordering $\prec$ of $\omega, t \in I, \prec \operatorname{sums} h^{x}(n)(t)=J_{x_{n}}^{h(n)}(t)$ in $B$, then

$$
\sum_{n \in \omega}^{\prec} h^{x}(n)(t)=\sum_{n \in C_{l}}^{\prec} h(n)=\sum_{C_{t}}^{\prec} h .
$$

Thus, $\prec \operatorname{sums} h^{x}(n)(t)$ for every $t \in I$ iff $\prec$ sums $h$ over C; that is, $\prec$ sums pointwise $h^{x}$ iff $\prec$ sums $h$ over C. Similarly, $\prec \operatorname{sums} h^{x}$ in $\operatorname{Reg}(I, B)$ -i.e., uniformly on $I$-iff $\prec$ sums $h$ uniformly over C.

We leave the easy proof of the following proposition to the reader. The nonbelievers are referred to Lemma 3 in $\$ 4$, that extends it.

Proposition 1. Let $h: \omega \rightarrow R$ be conditionally summable. Then there is a dense sequence $x$ in I such that $h$ is $x$-conditional.

If $\prec$ sums $h$ over $\mathrm{C}$, then a sum-function $\stackrel{\prec}{s}$ is defined over $\mathrm{C}$ by:

$$
s(C)=\sum_{C}^{\prec} h \quad(C \in \mathrm{C}) .
$$

We are now ready to eliminate $h^{x}$ from the statement of Theorem 3:

Theorem 3'. Let $B=R$. Let $h: \omega \rightarrow B$ be conditionally summable. Then there is an I-chain $\mathrm{C}=\left\{C_{t}: t \in I\right\}$ such that:

(i) There is an w-ordering $\prec_{0}$, that sums $h$ uniformly over C. Set

$$
s_{0}(t)=s_{s}^{\gamma_{0}}\left(C_{t}\right) \quad(t \in I) .
$$

(ii) For every continuous $g$ : $I \rightarrow R$ with $g(0)=0$ there is an w-ordering $\prec$ 
that sums $h$ uniformly over $C$, satisfying for every $t \in I$ :

$$
\grave{s}\left(C_{t}\right)=s_{0}(t)+g(t) \text {. }
$$

(iii) For every $\omega$-ordering $\prec$ of $\omega$ that sums $h$ uniformly over $C$ there is a continuous $g: I \rightarrow R$ satisfying $g(0)=0$ such that for every $t \in I$

$$
\varsigma s\left(C_{t}\right)=s_{0}(t)+g(t) \text {. }
$$

It is easy to derive Theorem 1 from (i) and (ii) as follows. Let $a \in R$. We have to show that for some $\omega$-ordering $\prec$ of $\omega$ we have $\sum_{\omega}^{\prec} h=a$. Let $b=a-s_{0}(1)$. Let $g(t)=t b$. By (ii) pick an $\omega$-ordering $\prec$ satisfying

$$
\stackrel{\prec}{s}\left(C_{t}\right)=s_{0}(t)+g(t) \text {. }
$$

Then

$$
\sum_{\omega}^{\prec} h=\prec s\left(C_{1}\right)=s_{0}(1)+g(1)=a .
$$

3. To generalize Theorem 3 we first extend the notion of being $x$ conditional to $h$ from $I$ into arbitrary Banach space $B$. Fix $h: \omega \rightarrow B$ and a dense sequence $x=\left(x_{n}\right)_{n \in \omega}^{\cdot}$ in $I$. Let $F \in[\omega]^{<\omega}$, and assume $F=$ $\left\{n_{1}, \ldots, n_{r}\right\}$, where $x_{n_{1}}<x_{n_{2}}<\cdots<x_{n_{r}}$. We shall say that $F$ is proper for $h$ with respect to $x$ if:

$$
\max _{1<i<r}\left\|\sum_{j=1}^{i} h\left(n_{j}\right)\right\|<2\|h(F)\| .
$$

Geometrically, $F$ is proper for $h$ w.r.t. $x$ if the polygonal line from 0 to $h(F)$ through $h\left(n_{1}\right), h\left(n_{1}\right)+h\left(n_{2}\right), h\left(n_{1}\right)+h\left(n_{2}\right)+h\left(n_{3}\right), \ldots$ never gets out or the ball centered at 0 and of radius $2\|h(F)\|^{3}$

We set $C_{t}=\left\{n: x_{n}<t\right\}$ for $t \in I$.

We say that $h: \omega \rightarrow B$ is $x$-conditional if $h$ satisfies (1) and:

(3) For every $0<a<b<1,\left\{h(F): F \in\left[C_{b}-C_{a}\right]^{<\omega}, F\right.$ is proper for $h$ w.r.t. $x$ \} is dense in $B$.

(1) and (3) are equivalent to (1) and (2) if $B=R$. An $x$-conditional $h$ : $\omega \rightarrow B$ exists if and only if $B$ is a separable Banach space.

A straightforward generalization of the proof of Theorem 3 gives [M, Theorem 5]:

THEOREM 4. Let $B$ be arbitrary Banach space and assume that $h: \omega \rightarrow B$ is $x$-conditional. Then there is an $s_{0} \in \operatorname{Reg}(I, B)$ such that

$$
S\left(h^{x}\right)=s_{0}+C(I, B) \text {. }
$$

\footnotetext{
${ }^{3}$ The choice of 2 here is somewhat arbitrary for the sequel. Any constant $>1$ could be chosen. See [M, 85].
} 
4. Let $B$ be arbitrary Banach space. Define a constant $K_{B}$ as the smallest $K$ such that for every finite sequence $\left(v_{1}, \ldots, v_{n}\right)$ in $B$ satisfying $v_{1}+\cdots+v_{n}$ $=0$, there is a rearrangement $\left(1^{\prime}, \ldots, n^{\prime}\right)$ of $(1, \ldots, n)$ such that

$$
\max _{1<i<n}\left\|\sum_{j=1}^{i} v_{j}\right\|<K \max _{1<i<n}\left\|v_{i}\right\| .
$$

Clearly, $1 \leqslant K_{B} \leqslant \infty$. In [B1] Bergström bases his proof to Theorem 2 on the following Lemma (found also in [S]):

LEMMA 1. Let $B$ be finite dimensional. Then $K_{B}<\infty$.

REMARK. 1. In [B2] Bergström shows that if $E^{d}$ denotes the $d$-dimensional Euclidean space, then $K_{E^{2}}=\sqrt{5} / 2$ and in general $K_{E^{d}} \sim \sqrt{d}$.

2. From Dvoretzky-Rogers' Theorem [D2] it follows that conversely, if $K_{B}<\infty$ then $B$ is finite dimensional.

The following lemma is equivalent to Lemma 1.

LEMMA 2. Let $B$ be finite dimensional Banach space. Then there is a finite $K$ such that whenever $v=v_{1}+\cdots+v_{n}$, there is a rearrangement $\left(1^{\prime}, \ldots, n^{\prime}\right)$ of $(1, \ldots, n)$ such that

$$
\max _{1<i<n} \min _{0<t<1}\left\|\sum_{j=1}^{i} v_{j^{\prime}}-t v\right\|<K \max _{1<i<n}\left\|v_{i}\right\| .
$$

The smallest such $K$ is $K_{B}$.

(Hint. Let $k \in \omega$ satisfy $\|v\|<k \cdot \max _{1<i<n}\left\|v_{i}\right\|$.

Set $u=(1-1 / k) v$ and apply Lemma 1 to the $(n+k)$-sequence $\left(v_{1}, \ldots, v_{n}, u, \ldots, u\right)$.)

Corollary. If $B$ is finite dimensional, $v=v_{1}+\cdots+v_{n},\left\|v_{i}\right\|<\|v\| / K_{B}$ then there is a rearrangement $\left(1^{\prime}, \ldots, n^{\prime}\right)$ of $(1, \ldots, n)$ such that

$$
\max _{1<i<n}\left\|v_{1^{\prime}}+\cdots+v_{i^{\prime}}\right\|<2\|v\| \text {. }
$$

Proof. Let $\left(1^{\prime}, \ldots, n^{\prime}\right)$ be given by Lemma 2 . Let $1<i<n$. Choose $0<t<1$ with

$$
\left\|v_{1^{\prime}}+\cdots+v_{i^{\prime}}-t v\right\|<K_{B} \cdot \max _{1<j<n}\left\|v_{j}\right\|<\|v\| .
$$

Then $\left\|v_{1^{\prime}}+\cdots+v_{i^{\prime}}\right\|=\left\|\left(v_{1^{\prime}}+\cdots+v_{i^{\prime}}-t v\right)+t v\right\|<2\|v\|$.

Proposminon 2. Let $B$ be arbitrary Banach space. Let $h: \omega \rightarrow B$ satisfy: $\left\{h(F): F \in[\omega]^{<\omega}\right\}$ is dense in $B$. Let $A \subseteq \omega$ satisfy $\Sigma_{n \in A}\|h(n)\|<\infty$. Then $\left\{h(F): F \in[\omega-A]^{<\omega}\right\}$ is dense in $B$.

(We shall need the proposition only for finite $A$.) 
Proof. Let $v \in B, \varepsilon>0$ be given. We shall find $F \in[\omega-A]^{<\omega}$ with $\|h(F)-v\|<\varepsilon$. Fix $n$ such that

$$
\sum_{n<m}\|h(m)\|<\frac{\varepsilon}{2} .
$$

Let $A^{\prime}=A \cap\{0, \ldots, n-1\}$. For $G \subseteq A^{\prime}$ define:

$$
V_{G}=\left\{h(F): F \in[\omega]^{<\omega}, F \cap A^{\prime}=G\right\} .
$$

Since $\left\{G: G \subseteq A^{\prime}\right\}$ is finite, and $\left\{h(F): F \in[\omega]^{<\omega}\right\}=\cup_{G \subseteq A^{\prime}} V_{G}$ is dense in $B$, there is a $G_{0} \subseteq A^{\prime}$ such that $V_{G_{0}}$ is dense in $B$. Choose $F \in[\omega-A]^{<\omega}$, $H \in\left[A-A^{\prime}\right]^{<\omega}$ such that $v_{1}=h\left(G_{0} \cup F \cup H\right)=h\left(G_{0}\right)+h(F)+h(H)$ satisfies $\left\|v_{1}-\left(v+h\left(G_{0}\right)\right)\right\|<\varepsilon / 2$. Then we have:

$$
\begin{aligned}
\|h(F)-v\| & =\left\|\left(h(F)+h\left(G_{0}\right)+h(H)\right)-\left(v+h\left(G_{0}\right)\right)-h(H)\right\| \\
& <\left\|v_{1}-\left(v+h\left(G_{0}\right)\right)\right\|+\|h(H)\|<\varepsilon / 2+\varepsilon / 2=\varepsilon .
\end{aligned}
$$

A corollary of Theorem 4 and the preceding remarks is

LEMMA 3. Let $B$ be finite dimensional. Let $h: \omega \rightarrow B$ satisfy (1) and

$$
\left\{h(F): F \in[\omega]^{<\omega}\right\} \text { is dense in } B \text {. }
$$

Then there is a dense sequence $x=\left(x_{n}\right)_{n \in \omega}$ in I such that $h$ is $x$-conditional.

Proof. Let $V$ be a countable dense set in $B$. Let $\left(v_{n}\right)_{n \in \omega}$ be an enumeration of $V$ such that for each $v \in V,\left\{n: v_{n}=v\right\}$ is infinite. Let $B_{n}=\{u \in B$ : $\left.\left\|u-v_{n}\right\|<1 /(n+1)\right\}$.

We construct the sequence $x$ in steps, defining in each step $x_{p}$ for $p$ in a fresh finite subset of $\omega$. Assume that $x_{p}$ is already defined for $p \in A$, where $A$ is a finite subset of $\omega$.

Step $2 k$. Let $\delta=\max \{\|h(n)\|: n \in \omega-A\}$. Let $F=\{n \in \omega-A:\|h(n)\|$ $=\delta$ \}. Since $h$ satisfies (1), $F$ is finite. Pick $x_{n} \in(0,1)$ for $n \in F$ so that $x_{n} \neq x_{p}$ for $p \in A$, and $x_{n} \neq x_{m}$ for $n \neq m(n, m \in F)$.

Step $2 k+1$. Let $I_{1}, \ldots, I_{r}$ be the components of $I-\left\{x_{p}: p \in A\right\}$. By Proposition 2, choose $F_{q} \in[\omega-A]^{<\omega}$ for $q=1, \ldots, r$ so that $q \neq q^{\prime}$ implies $F_{q} \cap F_{q^{\prime}}=\varnothing$ and $h\left(F_{q}\right) \in B_{k^{\prime}}$.

Let $F_{q}=\left\{n_{1}, \ldots, n_{k}\right\}$ where the indices are chosen so that for $i=$ $1, \ldots, k$ we have:

$$
\left\|h\left(n_{1}\right)+\cdots+h\left(n_{i}\right)\right\|<2\left\|h\left(F_{q}\right)\right\| .
$$

This is possible by the cor dary to Lemma 2. Choose $x_{n_{1}}<x_{n_{2}}<\cdots<x_{n_{k}}$ in $I_{q}$, dividing $I_{q}$ into intervals of equal length.

It is easy to check that $\left(x_{n}\right)_{n \in \omega}$ enumerates a dense set in $I$ with no repetitions (Step $2 k$ makes sure that $x_{n}$ is defined for every $n \in \omega$ ).

Given $0<a<b<1$ there is a $k_{0}$ such that for every $k>k_{0}$ one of the 
intervals $I_{q}$ considered in Step $2 k+1$ is included in $(a, b)$, and so for some $F \in\left[C_{b}-C_{a}\right]^{<\omega}, F$ is proper for $h$ w.r.t. $x$ and $h(F) \in B_{k}$. It follows that $h$ is $x$-conditional.

REMARK. By an easy modification one shows that given any countable dense set $X$ in $I$, the dense sequence $x=\left(x_{n}\right)_{n \in \omega}$ of Lemma 3 can be so chosen that $X=\left\{x_{n}: n \in \omega\right\}$.

Combining Lemma 3 and Theorem 4 we have:

Theorem 5. Let $B$ be finite dimensional. Let $h: \omega \rightarrow B$ satisfy (1) and (4). Then there is a dense sequence $x$ in $I$, and an $s_{0} \in \operatorname{Reg}(I, B)$ such that:

$$
S\left(h^{x}\right)=s_{0}+C(I, B) \text {. }
$$

5. We combine now Theorem 5 with Steinitz's ideas to generalize Theorem 2 as follows.

TheOREM 6. Let $B$ be finite dimensional, and let $h: \omega \rightarrow B$ be conditionally summable. Then there is a subspace $N$ of $B$ of dimension $>0$, a dense sequence $x$ in $I$, and an $s_{0} \in \operatorname{Reg}(I, B)$ such that:

$$
S\left(h^{x}\right)=s_{0}+C(I, N) .
$$

Proof. It follows from Steinitz [S] that there are subspaces $M, N$ of $B$ such that $B=M \oplus N$, and if $h_{M}: \omega \rightarrow M, h_{N}: \omega \rightarrow N$ are determined by the equations $h(n)=h_{M}(n)+h_{N}(n), h_{M}(n) \in M, h_{N}(n) \in N$, then

(i) $\sum_{n \in \omega}\left\|h_{M}(n)\right\|<\infty$,

(ii) $S\left(h_{N}\right)=N$.

(ii) implies that $h_{N}$ satisfies (1) and that $\left\{h_{N}(F): F \in[\omega]^{<\omega}\right\}$ is dense in $N$. By Theorem 5 we pick a dense sequence $x=\left(x_{n}\right)_{n \in \omega}$ in $I$ and $s_{2} \in \operatorname{Reg}(I, N)$ such that:

$$
S\left(h_{N}^{x}\right)=s_{2}+C(I, N) .
$$

Since $\left\|h_{M}^{x}(n)\right\|=\left\|h_{M}(n)\right\|$, we have $\Sigma_{n \in \omega}\left\|h_{M}^{x}(n)\right\|<\infty$. Thus, there is an $s_{1} \in \operatorname{Reg}(I, M)$ such that every $\omega$-ordering $\prec$ of $\omega$ sums $h_{M}^{x}$ to $s_{1}$ in $\operatorname{Reg}(I, M)$.

Since $h^{x}(n)=h_{M}^{x}(n)+h_{N}^{x}(n)$, we see that an $\omega$-ordering $\prec$ sums $h^{x}$ iff it sums $h_{N}^{x}$, and whenever $\prec$ sums $h_{N}^{x}$ to $f$ it sums $h^{x}$ to $s_{1}+f$.

Let $s_{0}=s_{1}+s_{2}$. Then $s_{0} \in \operatorname{Reg}(I, B)$ and we have:

$$
S(h)=s_{1}+S\left(h_{N}\right)=s_{1}+\left(s_{2}+C(I, N)\right)=s_{0}+C(I, N) .
$$

We restate Theorem 6, using the lexicon of $\$ 2$ :

Theorem 6'. Let $B$ be finite dimensional Banach space. Let $h: \omega \rightarrow B$ be conditionally summable. Then $B$ has a subspace $N$ of dimension $>0$, and there is an I-chain $\mathrm{C}=\left\{C_{t}: t \in I\right\}$ of subsets of $\omega$ so that: 
(i) There is an w-ordering $\prec_{0}$ that sums $h$ uniformly over C. Let

$$
s_{0}(t)=\stackrel{<}{s}^{\gamma_{0}}\left(C_{t}\right) \quad(t \in I) .
$$

(ii) For every continuous $g: I \rightarrow N$ satisfying $g(0)=0$ there is an $\omega$-ordering $\prec$ that sums $h$ uniformly over $\mathrm{C}$, satisfying for every $t \in I$

$$
\stackrel{\prec}{s}\left(C_{t}\right)=s_{0}(t)+g(t) \text {. }
$$

(iii) For every w-ordering $\prec$ that sums $h$ uniformly over $\mathrm{C}$ there is a continuous $g: I \rightarrow N$ satisfying $g(0)=0$, such that for every $t \in I$

$$
\prec\left(C_{t}\right)=s_{0}(t)+g(t) \text {. }
$$

Acknowledgement. We are grateful to Paul Erdös for bringing Steinitz's work to our attention, and to Arie Dvoretzky for several helpful conversations.

\section{REFERENCES}

[B1] V. Bergström, Eine neuer Beweis eines satzes von E. Steinitz, Hamburgische Abhandlungen 8 (1930), 148-152.

[B2] 205-219. , Zwei Sätze über ebene Vectorpolygone, Hamburgische Abhandlungen 8 (1930),

[D1] V. Drobot, Rearrangements of series of functions, Trans. Amer. Math. Soc. 142 (1969), 239-248.

[D2] A. Dvoretzky and C. A. Rogers, Absolute and unconditional convergence in normed linear spaces, Proc. Nat. Acad. Sci. U.S.A. 36 (1950), 192-197.

[G] C. Goffman, G. Moran and D. Waterman, The structure of regulated functions, Proc. Amer. Math. Soc. 57 (1976), 61-65.

[H] T. H. Hildebrandt, On unconditional convergence in normed vector spaces, Bull. Amer. Math. Soc. 46 (1940), 959-962.

[L] P. Levy, Sur les séries semi-convergentes, Nouv. Ann. Math. 64 (1905), 506-511.

[M] G. Moran, Of regulated and steplike functions, Trans. Amer. Math. Soc. 231 (1977), 249-257.

[O] W. Orlicz, Beiträge zur Theorie der orthogonal Entwicklung. II, Studia Math. 1 (1929), 242.

[S] E. Steinitz, Bedingt Konvergente Reihen und konvexe Systeme, J. Mathematik 143 (1913), 128-175.

DePARTMENT OF MATHEMATICS, UNIVERSITY OF HAIFA, HAIfA, ISRARL 